\title{
The rate and locus of development of rat vallate taste buds
}

\author{
Bruce Oakley, Douglas E. LaBelle, Robert A. Riley, Kristen Wilson and Lan-Hsin Wu \\ Biology Department, University of Michigan, Ann Arbor, MI 48109 (U.S.A.)
}

(Accepted 23 October 1990)

Key words: Chorda tympani; Induction; Maturation; Regeneration; Sensitive period; Stem cell; Ninth nerve

\begin{abstract}
Several hundred taste buds develop and mature in the trench walls of the rat's vallate papilla during the first 3 months after birth. The objective of this investigation of taste bud development was to determine: (i) whether the vallate papilla has local growth zones where new taste buds form, (ii) whether new taste buds arise by the division of mature taste buds, (iii) how many days are required for a new taste bud to mature, and (iv) whether a taste pore forms as the taste bud reaches a critical volume. Camera lucida drawings were made of more than 4000 iron hematoxylin-stained, serially sectioned, vallate taste buds. The relative abundance of immature taste buds declined exponentially with age, from $18 \%$ of the mature taste buds at day 15 to $2 \%$ at day 90 . At days 21,33 and 45 most of the immature taste buds (those lacking a taste pore) were located in growth zones at the anterior and posterior extremes of the vallate trench. A mean of 10.5 days was required for the maturation of each cohort of immature taste buds present at days 15,21, 33 and 45 . Vallate taste buds were added de novo; fission of mature taste buds was rare. Taste buds varied widely in the taste bud volume at which a pore formed and in the final volume of the taste bud.
\end{abstract}

\section{INTRODUCTION}

The rat's tongue has a single vallate papilla that lies on the dorsal midline. The vallate papilla consists of a $1 \mathrm{~mm}$ diameter island of tissue nearly surrounded by an elliptical trench. The several hundred taste buds in the vallate trench walls mature during a postnatal developmental period lasting from birth to 90 days $^{6}$. A similar developmental time course has been shown for hamster vallate taste buds ${ }^{11}$. Developing taste buds appear to be sealed off until a taste pore forms to expose the apical tips of taste receptor cells to chemical stimulants in the oral environment. Since timed examinations of regenerating fungiform taste buds in gerbils suggested that the reemergence of the taste pore was the final step that restored functional taste responses ${ }^{4}$, we define a mature taste bud as one that has developed a taste pore. This definition of taste bud maturity does not exclude maturational changes that may continue after a pore has formed.

Recent studies have shown that the vallate taste buds of the rat are neurally induced ${ }^{7}$ during a sensitive period that is maximal from 0 to 10 days postpartum ${ }^{8}$. Over a 2 month postnatal period, mature vallate taste buds increase in length and width and display a 3-fold increase in cell number ${ }^{6}$. The developmental properties of immature taste buds represent the focus of the present work, since they have not been examined in detail in prior studies.
Measurement of the number and volume of immature taste buds at different neonatal ages, and determination of the spatial distribution of taste buds along the rostrocaudal axis of the vallate papilla allowed us to evaluate several issues in taste bud development. We sought: (i) to locate possible growth sites in the vallate papilla where the new taste buds might be formed, (ii) to determine how many days it takes for a new taste bud to mature, (iii) to determine whether a taste pore forms as the taste bud reaches a critical volume, and (iv) to evaluate whether developing taste buds only arise de novo, or also routinely arise by the division of mature taste buds.

\section{MATERIALS AND METHODS}

The vallate papilla was examined at $0,1,3,10,15,21,33,45$ and 90 days postnatally in Sprague-Dawley albino rats of both sexes (Rattus norvegicus; from Harlan, Indianapolis, IN). The animals were euthanized with an overdose of sodium pentobarbital and the vallate papilla excised and fixed by immersion for one week in a solution of $10 \%$ formalin, $15 \%$ sucrose and $1 \% \mathrm{NH}_{4} \mathrm{OH}$. Following fixation, the tissue was dehydrated through a graded ethanol series, cleared in xylene, and embedded in paraffin. Serial, $10-\mu \mathrm{m}$-thick, transverse sections were stained with Heidenhain's iron-hematoxylin ${ }^{6}$. On the basis of comparison with cryostat sections of fresh tissue, a factor of 1.27 was used to correct the dimensions for the shrinkage produced by tissue processing.

In 15 to 19-day-old animals, camera lucida drawings were made of every taste bud profile found in each $10-\mu \mathrm{m}$ section of the vallate papilla. Over 15,000 profiles were drawn. An average mature taste

Correspondence: B. Oakley, Biology Department, Natural Science Building, University of Michigan, Ann Arbor. MI 48109, U.S.A. 
bud appeared in 3 successive $10-\mu \mathrm{m}$ sections, whereas its $4-6-\mu \mathrm{m}$ diameter taste pore was restricted to one $10-\mu \mathrm{m}$ section. Immature taste buds were defined as those buds whose profiles lacked a taste pore. By comparing drawings of adjacent sections it was possible to identify all mature and immature taste buds and assign an identifying number to each bud. The spatial distribution of mature and immature taste buds along the rostral-caudal axis of the vallate papilla was plotted in $30-\mu \mathrm{m}$ steps. The exact location of each taste bud on the rostral-caudal axis was specified by the position of its largest profile (center of mass). We used a graphics tablet and traced the area of each taste bud profile, summed the profile areas for each taste bud, and multiplied by the $10-\mu \mathrm{m}$ section thickness to obtain the volume of each taste bud, as corrected for tissue shrinkage. For each mature and immature taste bud we assigned an identifying number and measured its volume and position in 3 animals at each of the following ages: $15,21,33,45$ and 90 days.

\section{RESULTS}

Mature taste buds were characterized by a taste pore (arrowheads in Fig. 1A,B). In addition, mature taste buds had fusiform cells that were aligned with the longitudinal axis of the taste bud. The mature taste buds' nuclei were situated in the basal portion of the bud (e.g. Fig. 1A), while the slender, aligned, apical extensions of the taste cells converged to the taste pore (double-ended arrows in Fig. 1). Mature taste buds viewed in oblique sections were resolved by the prominent darkly stained taste pit enclosing the taste pore and by the absence of
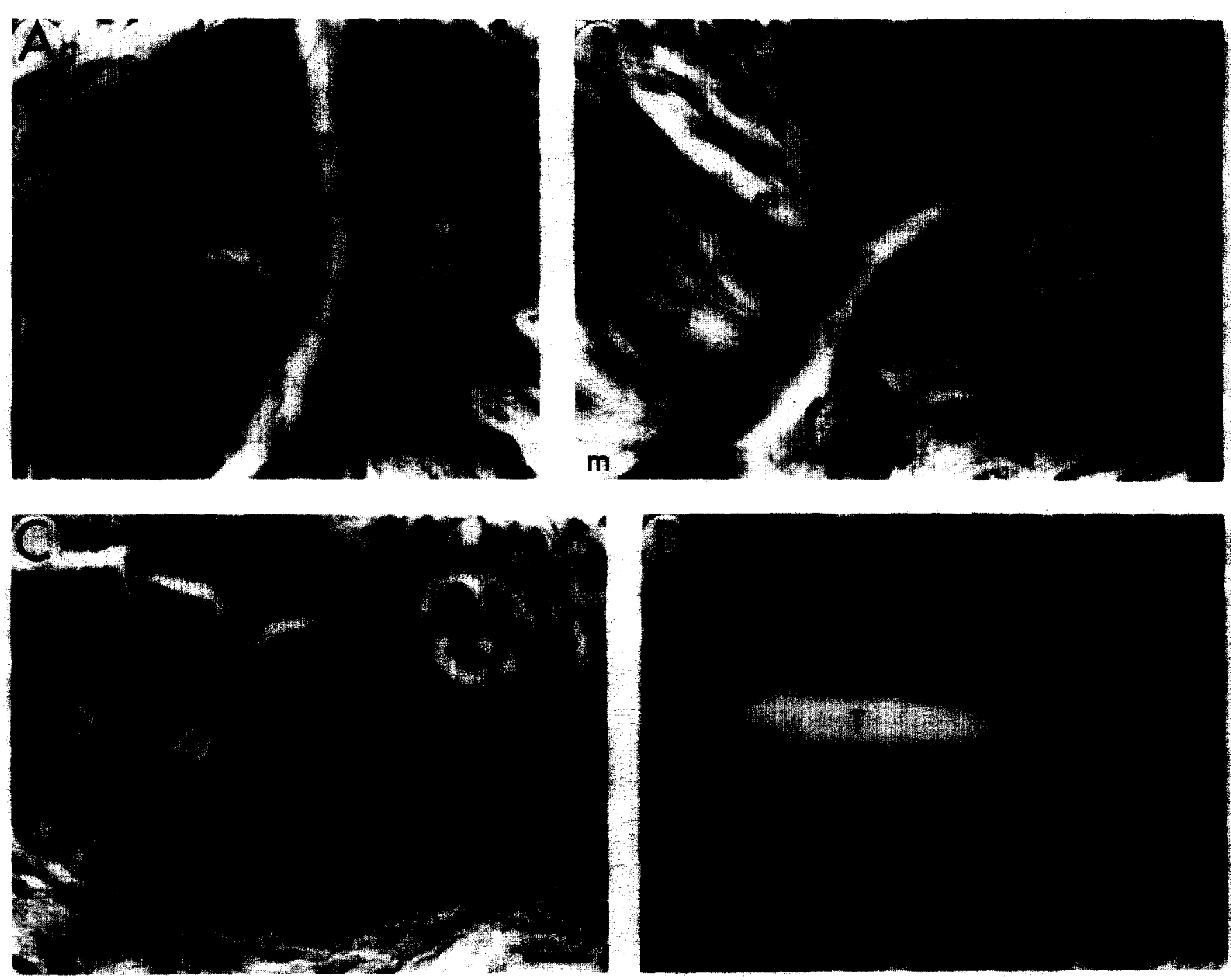

Fig. 1. A: a mature taste bud has a taste pore (arrowhead) leading to the trench (T). The elongated taste cells are parallel to the main axis of the taste bud. The apical extensions of these fusiform cells form a convergent palisade-array free of nuclei (double-ended arrow). In an oblique section, the nucleus-free apical cell extensions and the more darkly stained taste pit are visible (arrow) (33-day-old-rat). B: mature taste buds (m) vary in size. Two taste pores (arrowheads) and the slender, apical extensions of the taste cells (double-ended arrow) are evident. This section through the maximal extent of two immature taste buds (white arrows) reveals the range of sizes, from an immature taste bud with several basally situated nuclei, elongated cells, but no taste pore to a small neighbor with 3-4 cells (33-day-old rat). C: a large, round, mature taste bud $(\mathrm{m})$ with its cells extended toward the taste pit (double-ended arrow) contrasts with two smaller immature taste buds (i) whose nuclei are distributed throughout the buds. The cells of one immature bud have begun to elongate (33-day-old rat). D: 6 immature taste buds (i) cluster about the caudal extension of the trench ( $T$ ) (21 day-old rat). The apical portion of a small mature taste bud has cellular extensions without nuclei (double-ended arrow). The scale line in C represents 13.5, 12, 13, and 10.5 $\mu \mathrm{m}$ for A, B, C and D, resp. Iron-hematoxylin. 
nuclei in the apex of the taste bud (arrow in Fig. 1A). No mature taste buds with multiple taste pores were observed.

The cells of immature taste buds lacked several differentiated characteristics of mature taste buds. Consequently, in addition to the absence of a taste pore, immature taste buds were identifiable from the following positive traits: most immature taste buds were small, had cells that were either rounded or moderately elongated, but unaligned, whose nuclei were located in both apical and basal areas (Fig. 1B-D). Because new taste buds were being generated continually, at any time from 10-60 days of age a given animal had immature taste buds at all stages of maturity. Contiguous immature taste buds displayed considerable disparity in size and in the degree of taste bud maturation (Fig. 1B-D). Large and quite small immature taste buds might be immediate neighbors (white arrows in Fig. 1B) or an immature bud with rounded cells might be close to a more differentiated immature bud with partially elongated cells (Fig. 1C). The immature taste buds of 15- to 45-day-old rats often occurred in clusters, were generally smaller than mature taste buds and commonly lay so deep within the epidermis that no immediate access to the trench was evident. In Fig. 1D 6 immature taste buds on day 21 were clustered about, but not connected to the narrow, oval-shaped, rostral extension of the vallate trench. Immature taste buds were most abundant at the rostrocaudal extremes of the vallate trench (Fig. 1D). Immature taste buds were evident at postnatal days $0-90$, although by day 90 an average of fewer than 10 immature taste buds were present among the $500+$ mature taste buds.

Two observers were in close agreement in their assignments of mature and immature taste buds based upon their independent camera lucida drawings of serial sections of 6 vallate papillae. An occasional cell cluster was faintly demarcated and appeared in only one histological section. Such clusters were not counted as immature taste buds in order to minimize false positives.

As animals aged, the relative number of immature taste buds decreased exponentially as a percentage of mature taste buds (exponential least squares best fit, $r=$ $+0.94, P<0.02$, Fig. 2). By day 90 less than $2 \%$ of all taste buds were immature, both because there were only a few immature taste buds and because the total number of mature taste buds had increased substantially from day 15 to day 90 (inset in Fig. 2).

Since the total population of mature taste buds increased by 129 between days 15 and 45 , mature taste buds must have accumulated at a rate of about $4 /$ day during this 30-day period. During this same 30-day period, an average of $40 \pm 8$ immature taste buds were present on any given day (mean \pm 1 S.D.). Hence, given a maturation rate of $4 /$ day, the youngest taste buds in a cohort of 40 immature taste buds would require 10 days to mature. A 10-day maturation time was borne out by specific evaluation of the taste bud maturation rates at each of 4 animal ages: 15, 21, 33, 45 days. For any given number of mature and immature taste buds present on day $\mathrm{X}$, the population growth curve for mature taste buds (inset in Fig. 2) indicates the additional days required by the immature cohort to mature. For example, from the normal rate of increase in the number of mature taste buds after day 15 we determined graphically that the mean cohort of 48 immature taste buds present at day 15 would have to mature within 9.3 days. Similarly, to maintain the normal rate of increase in the number of mature taste buds, the cohort of 44 immature taste buds present at day 21 would have to mature in 10.7 days, the 38 immature buds at day 33 in 11.5 days and the 29 immature buds at day 45 in 10.7 days. Hence, the average rate of maturation of the cohorts of immature taste buds present at days $15,21,33$ and 45 was $10.5 \pm 0.9$ days (mean \pm 1 S.D.). While it was more difficult to evaluate taste bud maturation rates before day 10 because the taste buds were small and harder to recognize, it was clear that maturation was faster in the youngest animals. In 8 neonates at days $0-3$ there was a mean of $22 \pm 9$ immature taste buds per animal, which, based on the rise in the population growth curve ${ }^{6}$, should have matured in about 3 days.

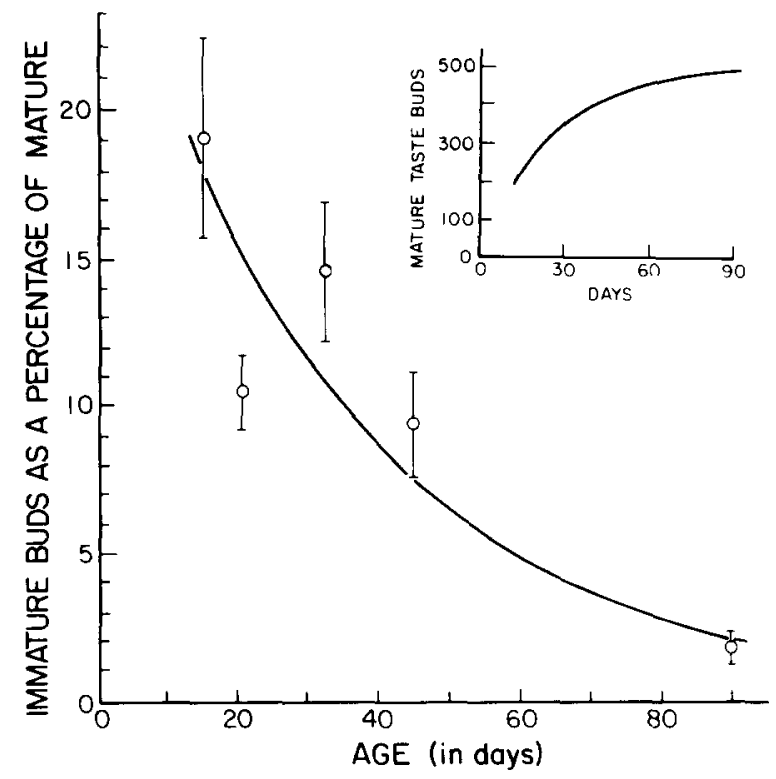

Fig. 2. In the rat vallate papilla the number of immature taste buds, as a percentage of mature taste buds, decreased exponentially from days 15 to 90 . The exponential curve is a least squares fit, $r=+0.94$. The open circles represent means \pm 1 S.D. of 3 animals at each age. The inset shows the increase in the number of mature vallate taste buds, as a function of postnatal age for these animals (third order, least squares best-fit polynomial, $r=+0.99$ ). 

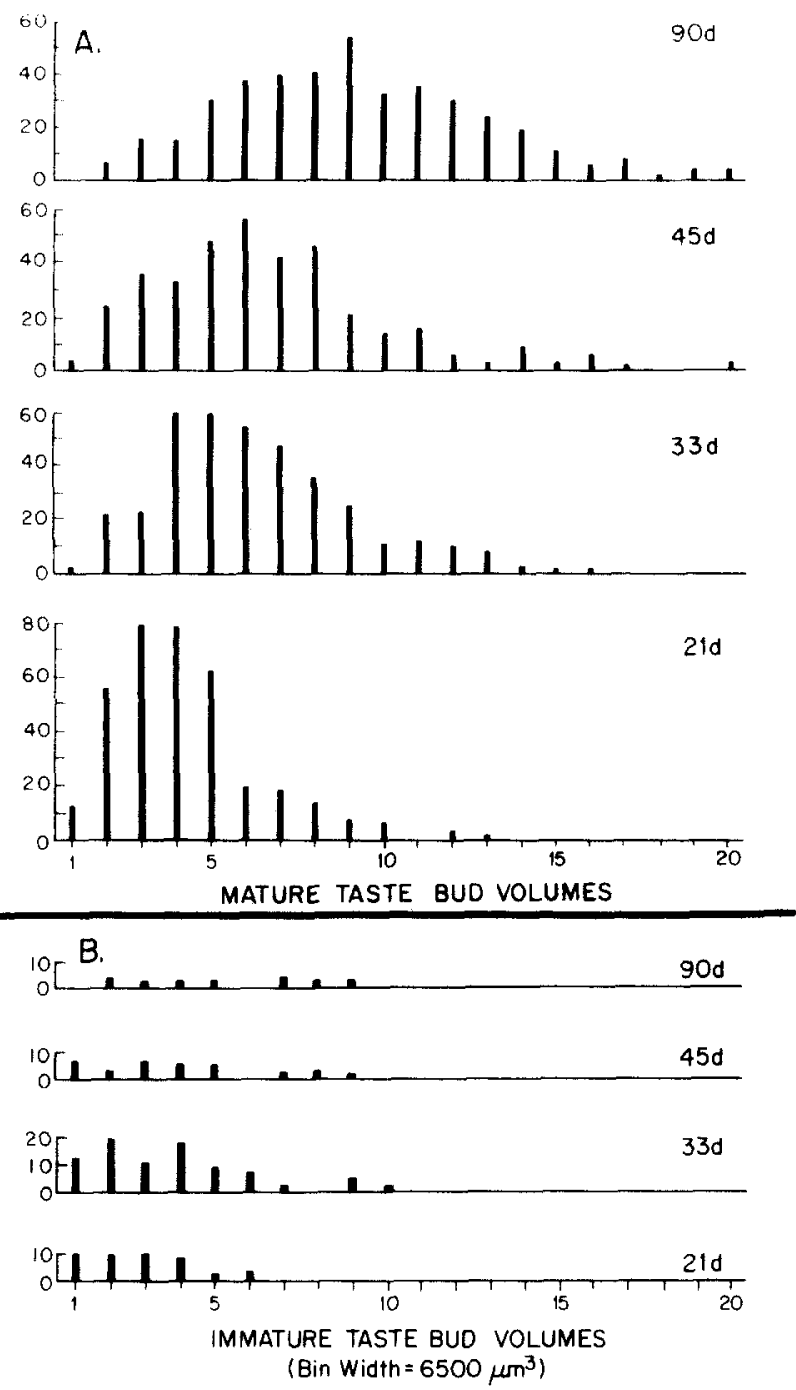

Fig. 3. The histograms show the frequency distribution of the volumes of vallate taste buds for representative individuals at days $21,33,45$ and 90 . For each histogram, bin 1 includes all taste buds with volumes less that $6500 \mu \mathrm{m}^{3}$. The $y$ axis gives the number of taste buds. A: volumes of mature taste buds in $\mathbf{4}$ individual animals at ages $21,33,45$ and 90 days. B: volumes of immature taste buds of the same 4 animals

For each animal aged 15-90 days we obtained histograms of volumes of mature and immature taste buds. Frequency histograms of mature taste bud volumes in 4 representative animals are shown in Fig. 3A. From days 21 to 90 there was a progressive increase in the average volume of mature taste buds and in the symmetry of their volume distribution. While individual bud volumes varied greatly at day 90 , as the largest taste buds were $10-20$ times bigger than the smallest, the volumes were rather symmetrically distributed. Although the mean immature taste bud was considerably smaller than the mean mature taste bud, the distributions of volumes overlapped substantially. That many mature taste buds were smaller than some immature taste buds argues against a set volume at which a pore is formed. The range of volumes

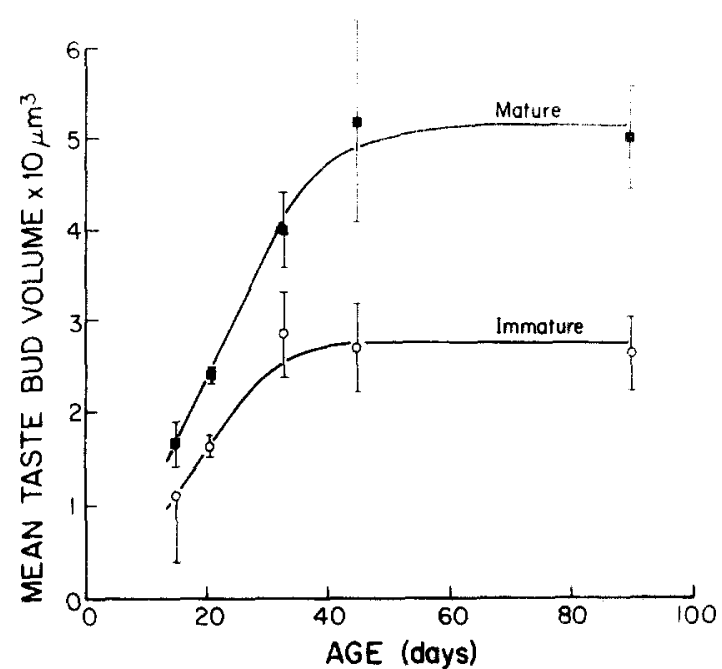

Fig. 4. The mean volume of both mature (filled circles) and immature taste buds (open circles) increases to a maximum as a function of animal age. (mean \pm 1 S.D., $n=3$ rats, except $n=2$, at day 15).

of immature taste buds did not change appreciably as animals aged (Fig. 3B), although fewer immature buds were present in older animals (45-90 days). When the mean taste bud volume is plotted as a function of age for all animals, it is evident that at every age the mature taste buds were larger on the average than the immature taste buds (Fig. 4). From days 15 to 90 the mean volume of immature taste buds increased from 10,900 to 26,600 $\mu \mathrm{m}^{3}$. Over the same period the mean volume of mature taste buds increased from 16,600 to $50,100 \mu \mathrm{m}^{3}$. The mean volume of mature taste buds rose more rapidly, then continued to rise to a maximum volume about two weeks later than for immature taste buds. The difference in volume between mature and immature taste buds and the increase in volume with age of both mature and immature taste buds are statistically significant $(P<$ $0.001, F=19.6$ and $P<0.004, F=5.5$, respectively).

To represent the spatial distribution of taste buds along the rostro-caudal axis of the vallate papilla, we plotted the number of mature taste buds and the number of immature taste buds present in each $30 \mu \mathrm{m}$ transverse slab of the papilla ( 3 serial $10-\mu \mathrm{m}$ sections). Most of the mature taste buds were found near the middle of the papilla with fewer mature buds at the rostral and caudal extremes of the papilla. In contrast, many immature taste buds were located in the caudal portion of the papilla at day 15 . On days 21,33 and 45 the immature taste buds were found predominantly at the rostral and the caudal extremes of the papilla. Fig. 5 shows the results for days 21 and 45 where the mature taste buds predominate in the center of the vallate papilla and the immature buds in the rostro-caudal extremes, which therefore constitute preferential developmental regions for new taste buds. 

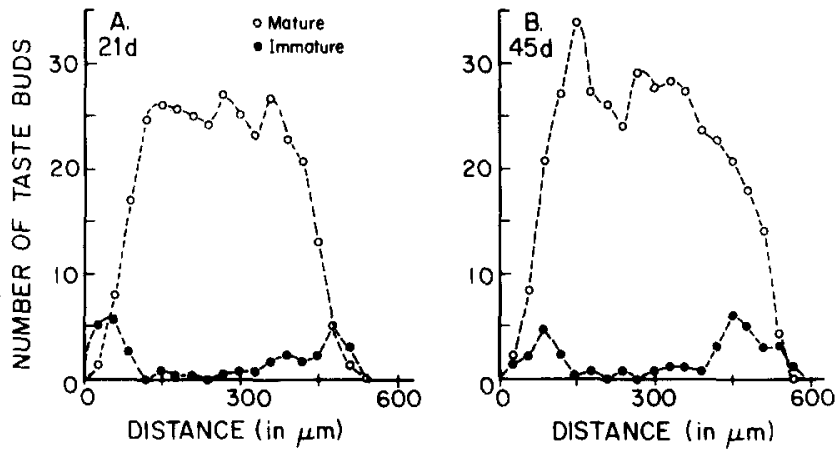

Fig. 5. The spatial distribution of mature taste buds (open circles) and immature taste buds (filled circles) is plotted along the rostro-caudal axis of the vallate papilla in $30-\mu \mathrm{m}$ increments which represent the sum of taste buds in sets of 3 adjacent $10-\mu \mathrm{m}$ sections. The dashed lines are second order polynomial interpolations between data points that are means of 3 21-day-old animals in (A) and 3 45-day-old animals in (B). The most rostral extent of the trench was set at $x=0$

This data on the rostro-caudal distribution of taste buds permits a spatial assessment of the previous calculations of taste bud maturation times in 15- to 45-day-old animals. If taste buds mature in 10-12 days, the spatial distribution of all taste buds present at a given age (mature + immature) should closely resemble the spatial distribution of only the mature taste buds 10-12 days later. For example, the spatial distribution of the immature and mature taste buds at day 33 was similar to the spatial distribution of mature taste buds at day 45 (Fig. 6A). This comparison, made over a 12-day interval, is quite close to the 11.5-day interval when the spatial distributions were predicted to be the same. Longer intervals were also examined for accuracy of the predicted spatial distribution. It is possible to approximate the 24-day interval between days 21 and 45 by combining maturation times for cohorts of immature taste buds present at day 21 and at day 33 (10.7 days +11.5 days $=$ a 22.7 -day interval). (Given that the immature taste buds at day 21 mature in 10.7 days, the immature taste buds present 12 days later at day 33 should represent a later, independent cohort of immature buds, and thus are not added twice.) There was good agreement between the spatial distribution of mature taste buds at day 45 and the curve resulting from the addition of the day 21 mature + immature taste buds with the day 33 immature taste buds (Fig. 6B). The best fit distributions are closely similar in height and position along the abscissa. The area under the composite curve $(21 \mathrm{~d} \mathrm{M}+\mathrm{I}+33 \mathrm{~d} \mathrm{I})$ is $99.9 \%$ of the area under the day 45 mature taste bud curve.

\section{DISCUSSION}

\section{Maturation time}

Camera lucida drawings allowed us to align serial sections of the densely packed vallate taste buds and discriminate mature taste buds with their elongated cells, basally situated nuclei and slender, convergent, apical cell extensions and taste pore from immature taste buds that characteristically lacked these traits. In 2- to 8week-old animals, immature taste buds matured in a
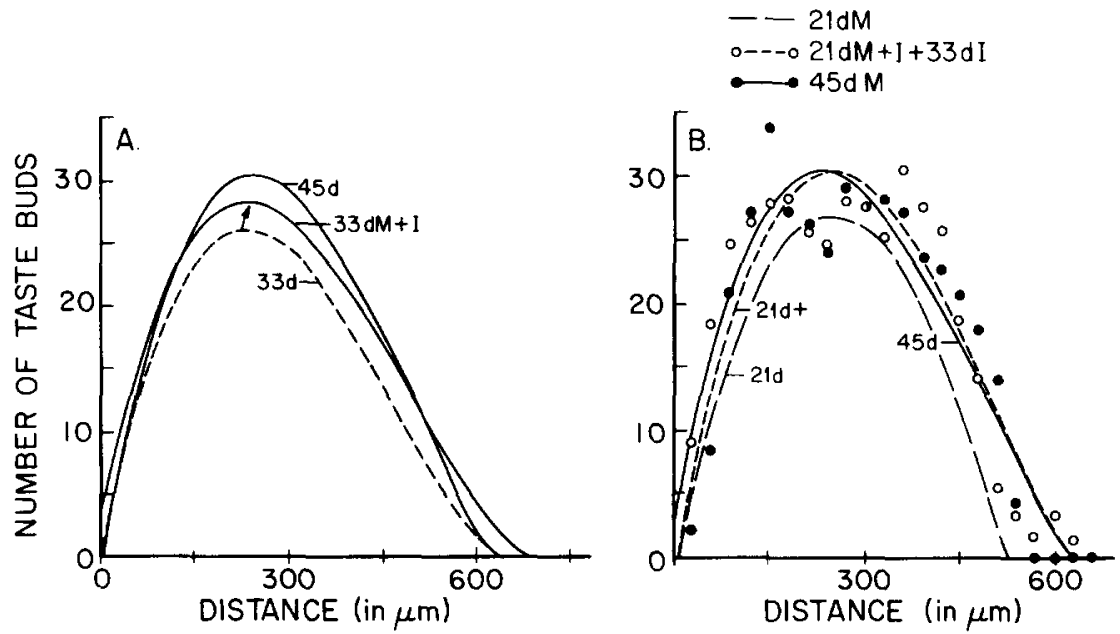

Fig. 6. The mean rostro-caudal spatial distribution of taste buds in the vallate papilla at several ages. A: the summed spatial distribution of mature and immature taste buds at day 33 (solid curve labeled 33d M+I) approximates the distribution of mature taste buds at day 45 (solid curve labeled $45 \mathrm{~d}$ ). The dashed curve is the spatial distribution of mature taste buds at $33 \mathrm{~d}$. B: the spatial distribution of mature taste buds at day 45 (solid curve and solid circles) is closely approximated by the sum of taste buds in three spatial distributions: mature + immature taste buds at day $21+$ immature taste buds at day 33 (short dashed curve labeled $21 d+$, open circles). At day 21 mature taste buds have a more restricted spatial distribution (curve with long dashes, labeled 21d). All spatial distributions of taste buds in A and B are represented by third order polynomial curves fitted by the least squares procedure to data points, each representing the mean of 3 animals. The correlation coefficients of the 6 fitted curves range from +0.89 to +0.97 . 
mean of 10.5 days. Accurate estimates of maturation times were not possible after day 60 , because the flatness of the curve characterizing the increase in numbers of mature taste buds (inset in Fig. 2) magnifies small errors in counting immature taste buds into large variations in the calculated maturation time for immature taste buds. In rats less than a week old, it was more difficult to resolve small cell clusters that might represent nascent taste buds; even taste buds with a pore were smaller and less differentiated than in older animals. Regardless of uncertainties in the exact number of immature taste buds present in newborns, it is likely that the few that were present matured in less than a week. Had the immature taste buds present in 0- to 3-day-old animals matured in 10.5 days, it would have meant that most of the $100+$ mature taste buds observed on day 10 were cryptically present at birth as immature taste buds. Since it is unlikely that so many immature taste buds escaped early detection, we conclude that in the youngest postnatal animals the taste bud maturation time is significantly shorter than 10.5 days; perhaps as short as 2-3 days. Given that mature taste buds of younger animals are smaller (Figs. 3 and 4) and have fewer cells ${ }^{6}$, it might require fewer cell cycles, and thus less time, to produce a mature taste bud in young neonates, especially if the cell cycle time is also shorter. We have observed a similar pattern of maturation times for rat foliate taste buds; about 4 days during the first postnatal week and 10 days by the second postnatal week (Oakley and Hosley, unpublished). In developing hamster epiglottis ${ }^{1}$, we calculate that the cohort of immature buds present on postnatal day 10 would mature in 9 days. Consequently, maturation times lasting for 9-10 days are probably not a peculiarity of rat vallate taste buds.

An analysis of the spatial distribution of rat vallate taste buds provided a partially independent confirmation of the validity of the 10.5 day maturation time. Addition of the spatial distributions of mature and immature taste buds generated a combined distribution that approximated the spatial distribution of mature taste buds one maturation cycle (Fig. 6A) or two maturation cycles (Fig. 6B) later. Taste buds probably remain near their birthplace, since significant taste bud migration in the trench would have distorted the spatial distribution curves.

The calculation of vallate taste bud maturation time assumes that each cohort matures in succession, i.e., that each taste bud matures in order of its birth. The accelerated maturation, or jumping ahead in the birthdate queue, of some immature taste buds would have been undetectable for that cycle, but by depleting the supply of immature taste buds, the next cohort would have had a shorter calculated maturation time. The relative constancy of the maturation time over days $15-45$
(10.5 \pm 0.9 days) suggests that serious breeches of birth-order maturation did not occur.

De novo taste bud formation vs fission of mature or immature taste buds

The first vallate taste buds necessarily form de novo. Subsequently, additional programs of taste bud development might emerge, allowing, for example, mature taste buds to arise by the division of large multi-pored mature taste buds ${ }^{2}$. We consider 4 alternatives to de novo formation: division of mature taste buds that are: (i) multi-pored or (ii) single-pored, and division of immature taste buds at the (iii) center or (iv) rostro-caudal extremes of the vallate papilla. (i) If a dual-pore mature vallate bud split apart into two single-pore mature buds, the process would have been directly detected only by capturing the moment of division. Rarely, a taste bud profile resembled the basal abutment of two crowded mature taste buds - or perhaps the ongoing fission of a dual-pored bud. However, profiles of about 4000 mature taste buds revealed no clear instance of an undivided mature bud with multiple pores. (ii) Alternatively, if immature buds originated by budding off from a singlepored mature taste bud, the budding process must have been displaced some nuclei apically and misaligned the cells, as these were two reliable characteristics of immature buds throughout the papilla. Further, immature taste buds predominated in the rostro-caudal extremes where there were fewer neighboring mature buds from which they might have arisen by division. It is paradoxical that immature taste buds were infrequent at later ages in those central regions replete with mature buds, if mature taste buds were an important added source of immature taste buds. Therefore, it seems that the division of mature taste buds, whether multi- or single-pored, was not a major source of vallate taste buds. (iii) Division of immature taste buds in the center of the papilla cannot be a commonplace event because immature taste buds in this location usually occurred singly, rather than in pairs. (iv) The division of immature taste buds in the rostrocaudal extremes of the papilla, remains a possibility. Fission may have occurred where immature taste buds were crowded together. In summary, while it is possible that some late developing taste buds arose by the fission of mature buds, this was probably a quantitatively unimportant source of taste buds, in comparison to de novo taste bud formation. Morphometric measurements have shown that the vallate trench continues to lengthen along the rostro-caudal axis from days 15 to 45 (ref. 6). Thus, the rostro-caudal extremes of the vallate trench may be considered zones of continued growth where developmental events, such as the de novo formation of taste buds, persist postnatally. 
Taste bud volume, pore formation and maturation time

An interesting feature of the volume distribution of immature and mature taste buds is that many immature buds were larger than the smaller mature buds (Fig. 3). This suggests that pore formation is not automatically triggered when an immature taste bud reaches a critical volume. (It is presumed, as already argued, that few mature taste buds became small secondarily by the division of larger, mature taste buds.) That taste buds mature (form a pore) at differing volumes does not necessitate the more rapid maturation of small taste buds. The volume of the taste bud should reflect the number of contributing stem cells, whereas the time for taste bud maturation might be correlated with the time required for a fixed number of cell divisions ${ }^{9,10}$. After taste pore formation, a mature bud enlarges by an increase in the length and the width of its cells and by the addition of more cells. From days 10 to 45 there is an average 3-fold increase in the number of cells per mature vallate taste bud ${ }^{6}$. Some taste buds might enlarge slowly and remain small, if only a few cycling stem cells contribute to their development (viz. day 90 panel in Fig. 3A).

\section{Differences between taste bud development and regener- ation}

Three lines of evidence suggest that the regeneration of taste buds probably does not recapitulate their development. First, in regeneration there is no parallel to the neural induction of taste buds ${ }^{7}$ and to the sensitive period in development, during which carefully timed denervation causes a failure of taste bud formation ${ }^{8}$, that

\section{REFERENCES}

1 Belecky, T.L. and Smith, D.V., Postnatal development of palatal and laryngeal taste buds in hamsters, J. Comp. Neurol., 293 (1990) 646-654.

2 Bradley, R.M., Cheal, M.L. and Kim, Y.H., Quantitative analysis of developing epiglottal taste buds in sheep, J. Anat., 130 (1980) 25-32.

3 Cheal, M.L. and Oakley, B., Regeneration of fungiform taste buds: temporal and spatial characteristics, J. Comp. Neurol., 172 (1977) 609-626.

4 Cheal, M.L., Dickey, W.P., Jones, L.B. and Oakley, B., Taste fiber responses during reinnervation of fungiform papillae, $J$. Comp. Neurol, 172 (1977) 627-646.

5 Farbman, A.I., Renewal of taste bud cells in rat circumvallate papillae, Cell. Tissue Kinet., 13 (1980) 349-357.

6 Hosley, M.A. and Oakley, B., Development of the vallate is irreversible even when crushed IXth nerve axons or the cross-regenerated chorda tympani nerve ${ }^{12}$ re-innervates the denervated vallate epithelium. We hypothesize that those taste bud progenitor cells that lack innervation at a critical time in development, fail to become taste bud stem cells and either degenerate or adopt other developmental fates. In adults, stem cells are defined as the permanent cell lines that survive denervation and taste bud degeneration. Because stem cells remain, reinnervation produces replacement taste buds promptly. Second, the development of many vallate taste buds requires about 10.5 days, whereas regeneration will occur in the 3-day interval between the arrival of regenerating axons in the epithelium and the reappearance of mature vallate taste buds $^{8}$. Similarly, it required about 3 days for fungiform taste buds to regenerate, open a taste pore, and function. The moment of fungiform taste bud maturation was established by recording the initial taste responses, after chorda tympani axons re-invaded the fungiform papillae in adult gerbils ${ }^{3,4}$. Evidently, taste bud regeneration can be several times faster than taste bud development. Third, development and regeneration differ in that one IXth nerve can maintain or cause the regeneration of about twice as many taste buds in adults as it can induce in neonatal development ${ }^{8}$. These differences probably reflect the more elaborate cellular commitments and transformations present during development of the sense of taste.

Acknowledgements. We are grateful to M.A. Hosley. This study was supported in part by NIH Grant DC-00083.

papilla and taste buds in rats, Anat. Rec,, 218 (1987) 216-222.

7 Hosley, M.A., Hughes, S.E. and Oakley, B., Neural induction of taste buds, J. Comp. Neurol., 260 (1987) 224-232.

8 Hosley, M.A., Hughes, S.E., Morton, L.L. and Oakley, B., A sensitive period for the neural induction of taste buds, $J$. Neurosci., 7 (1987) 2075-2080

9 Hume, W.J., Stem cells in oral epithelia. In C.S. Potten (Ed.), Stem Cells: Their Identification and Characterization, Churchill Livingston, NY, 1980, pp. 233-270.

10 Hume, W.J. and Potten, C.S., The ordered columnar structure of mouse filiform papillae, J. Cell Sci., 22 (1976) 149-160.

11 Miller Jr., I.J. and Smith, D.V., Proliferation of taste buds in the foliate and vallate papillae of postnatal hamsters, Growth, Dev. Aging, 52 (1988) 123-131.

12 Oakley, B., Taste bud development in rat vallate and foliate papillae. In P. Hnik, T. Soukup, R. Vejsada and J. Zelena (Eds.), Mechanoreceptors, Plenum, New York, 1988, pp. 17-22. 